\title{
Action of Winds Inside and Outside of Star Clusters
}

\author{
Jan Palouš ${ }^{1}$, Jim Dale ${ }^{1}$, Richard Wünsch ${ }^{1}$, \\ and \\ Sergiy Silich ${ }^{2} \&$ Guillermo Tenorio-Tagle ${ }^{2}$ \\ and \\ Anthony Whitworth ${ }^{3}$ \\ ${ }^{1}$ Astronomical Institute, ASCR \\ BočníII 1401, CZ-141 31, Prague 4, Czech Republic \\ email: palous@ig.cas.cz \\ ${ }^{2}$ Instituto Nacional de Astrofísica Optica y Electrónica, \\ AP 51, 72000 Puebla, Mexico \\ email: silich@inaoep.mx \\ ${ }^{3}$ School of Physics and Astronomy, Cardiff University, Queens Building, The Parade, Cardiff, \\ CF24 3AA, UK \\ email: ant@ac.uk
}

\begin{abstract}
The feedback form pre-main sequence and young stars influences their vicinity. The stars are formed in clusters, which implies that the winds of individual stars collide with each other. Inside of a star cluster, winds thermalize a fraction of their kinetic energy, forming a very hot medium able to escape from the cluster in the form of a large-scale wind. Outside of the cluster, the cluster wind forms a shock front as it interacts with the ambient medium which is accreted onto the expanding shell. A variety of instabilities may develop in such shells, and in some cases they fragment, triggering second generation of star formation. However, if the cluster surpasses a certain mass (depending on the radius and other parameters) the hot medium starts to be thermally unstable even inside of the cluster, forming dense warm clumps. The formation of next generations of stars may start if the clumps are big enough to self-shield against stellar radiation creating cold dense cores.
\end{abstract}

Keywords. stars: formation, ISM: structure, globular clusters: general, open clusters and associations: general

\section{Introduction}

The star formation in galaxies transforms ISM to stars, which at the same time return a fraction of their mass to the ISM. The gas recycling redistributes the yield of stellar evolution causing the chemical evolution of galaxies. Star formation is an important process of internal galactic evolution, however, in some cases, it may be triggered by galaxy external agents.

Stars are formed in groups in cold, dense cores of molecular clouds. The feedback of young and massive stars influences their vicinity by radiation, stellar winds and by supernova explosions. In this contribution we shall separately discuss the actions of winds, and of other contributors to the feedback, outside and inside of stellar clusters. The feedback in star forming regions drives turbulence, and it may trigger the next generation of star formation in the compressed shells of the ISM outside of star clusters, or it may initiate star formation in warm clumps inside massive and sufficiently compact young stellar clusters. 


\section{Outside of a star cluster}

The combined feedback of many stars in clusters forms expanding dissociation, ionization and shock fronts creating bubbles and expanding shells in the ISM. The shells are observed in HI in the Milky Way and many nearby galaxies. Radio surveys of the Northern (Hartmann \& Burton, 1997) and Southern (McClure-Griffiths et al., 2002) skies discovered hundreds of shells and shell-like structures on the size scales $10 \mathrm{pc}-1 \mathrm{kpc}$. Ehlerová \& Palouš (2005) have used an automated search algorithm to find more than 600 shells in the outer Milky Way. Observation of HI shells in external galaxies are summarized by Walter \& Brinks (1999), many more discoveries are expected in THINGS: the HI nearby galaxy survey (Walter et al., 2008). In the infrared, Churchwell et al. (2006) and Churchwell et al. (2007) found many 0.1 - 10 pc size shells in the GLIMPSE survey covering only a part of the plane of the Milky Way. Deharveng et al. (2003), Zavagno et al. (2006), Sidorin (2008), and Deharveng et al. (2009) identified shells observationally in optical, infrared, radio and other wavebands, some of them appear to have triggered star formation at their borders. Watson et al. (2009) analyzed some of the shells from the GLIMPSE survey to determine properties of YSOs related to them.

The observed shells seem to be created by the feedback from young and massive stars in clusters, following the "collect and collapse" scenario proposed by Elmegreen \& Lada (1977). It assumes that the massive stars of a star cluster supply energy into the interstellar medium as stellar winds and radiation creating an expanding bubble filled with a high temperature gas. The bubble expands due to its internal overpressure, sweeping the ambient medium into a dense shell. When the shell cools down it becomes gravitationally unstable forming fragments that are seeds of a new stellar generation.

Gravitational fragmentation of expanding shells has been studied under the assumption that their thickness is infinitesimally small. Vishniac (1983) derived the dispersion relation using a decomposition into spherical harmonics. With the linear analysis of hydrodynamical and Poisson equations in 2 dimensions Elmegreen (1994) and Whitworth et al. (1994) derived very similar dispersion relations composed of two stretching terms depending on the general expansion velocity of the shell $V_{s h}$ and on its radius $R_{s h}$ plus a term due to shell internal pressure depending on the speed of sound inside of the shell $c_{s h}$, which are opposed by the gravity term depending on the shell surface density $\Sigma_{s h}$.

$$
\omega(l)=-\frac{A V_{s h}}{R_{s h}}+\sqrt{\frac{B V_{s h}^{2}}{R_{s h}^{2}}-\frac{c_{s h}^{2} l^{2}}{R_{s h}^{2}}+\frac{2 \pi G \Sigma_{s h} l}{R_{s h}}},
$$

where $G$ is the gravitational constant and $(A, B)=\left(\frac{3}{2}, \frac{1}{4}\right)$ or $(3,1)$ for non-accreting or accreting shells, $l$ is the wave number and $\omega$ gives the growth rate of a perturbation. From this dispersion relation it is visible that initially, when the radius of the shell is small and its expansion velocity is large, the first term dominates and the shell is stable; we may say that it is stabilized by expansion. Later, when the shell radius becomes large and its expansion is decelerated, the first two terms are negligible. The time $t_{b}$, when it becomes unstable for the first time can be derived provided the functions $R_{s h}(t), V_{s h}(t)$ and $\Sigma_{s h}(t)$ are known. Then, there is a certain range of wave numbers given by the dispersion relation, that are unstable, and we may ask how quickly they grow, and try to construct the mass spectrum of fragments.

A shell expanding due to internal overpressure into a higher density medium is subjected to instabilities: initially, when the expansion velocity is large, the expansion driven by high internal thermal pressure is opposed by ram pressure decelerating the shell. However, the thermal pressure acts isotropically and the ram pressure is always directed 
against expansion, which creates, in the case of a small deviation from sphericity, a pressure component parallel to the shell surface leading to growth of perturbations, which is described as the Vishniac instability (Vishniac, 1983). Another instability appears when a low-density gas accelerates the high-density gas. This Rayleigh-Taylor instability, which forms long 'fingers' of high-density fluid extending to the low-density medium, is particularly important for large density differences. Later, when Vishniac and Rayleigh-Taylor instabilities combine with the Jeans gravitational instability, the shell breaks into fragments.

In order to see the effect of individual instabilities, in a series of papers (Dale et al., 2009; Wünsch et al., 2010; and Dale et al., 2010) we try to isolate the gravitational instability. Therefore, we analyze the shells expanding into a medium of very low density and set the internal pressure equal to the external one. The lack of effective gravitational/inertial force (the shell evolves ballistically) eliminates the Rayleigh-Taylor instability. At the same time, the negligible ram pressure eliminates the Vishniac instability. We explore with 3 dimensional hydrodynamical simulations the gravitational instability during the ballistic motion of expanding shells.

The value of external and internal pressures is essential, since it influences the shell thickness: at low pressures the shell thickness increases during its ballistic motion, which makes the short wavelengths more stable, compared to dispersion relation 2.1, the low mass fragments do not form in this case. On the other hand, at high pressures, when the shell thickness decreases, even shorter wavelengths become unstable, compared to dispersion relation 2.1, producing an excess of low mass fragments. We also give the thick shell dispersion relation and propose a new Pressure Assisted Gravitational Instability (PAGI), which involves the external pressures and explains the above described behavior. Finally, we follow the evolution of individual fragments: their mass distribution corresponds to the PAGI dispersion relation only at the beginning of fragmentation during its linear phase. Later, they accumulate the remaining mass of the shell in a strongly nonlinear process, which we call oligarchic accretion: the old fragments that were created initially accrete most of the mass and they leave only a small portion for others that were formed later.

As a next step, we shall also include the mass accumulation during its expansion, thus we shall be able to study other instabilities and explore the PAGI dispersion relation and oligarchic accretion in more general situations.

\section{Inside of a star cluster}

The young and massive stars in clusters deposite energy and mass into the intracluster medium. It happens in the form of radiation, winds and supernovae composing the stellar feedback in star clusters. They range from open clusters $(\mathrm{OCl})$ of $10^{4} M_{\odot}$ to globular clusters (GC) and super star clusters (SSC) of $10^{6} M_{\odot}$. We also consider the compact dwarf galaxies (CDG), which may be the most massive compact stellar groups, of mass up to $10^{8} M_{\odot}$. The energy deposited by individual young stars thermalizes in wind wind collisions causing a large overpressure inside of the cluster driving the large-scale star cluster wind. In the adiabatic solution of Chevalier \& Clegg (1985) the overpressure results in the wind blowing from the center to the periphery of the cluster where it reaches the sound speed $c_{s}$. Outside of the cluster, the adiabatic wind accelerates up to a velocity of $2 c_{s}$.

In a series of papers Tenorio-Tagle et al. (2007), Wunsch et al. (2007), Wunsch et al. (2008), Tenorio-Tagle et al. (2010), Silich et al. (2010), and Hueyotl-Zahuantitla et al. (2010) we describe a model where the stellar wind's mechanical energy is thermalized 


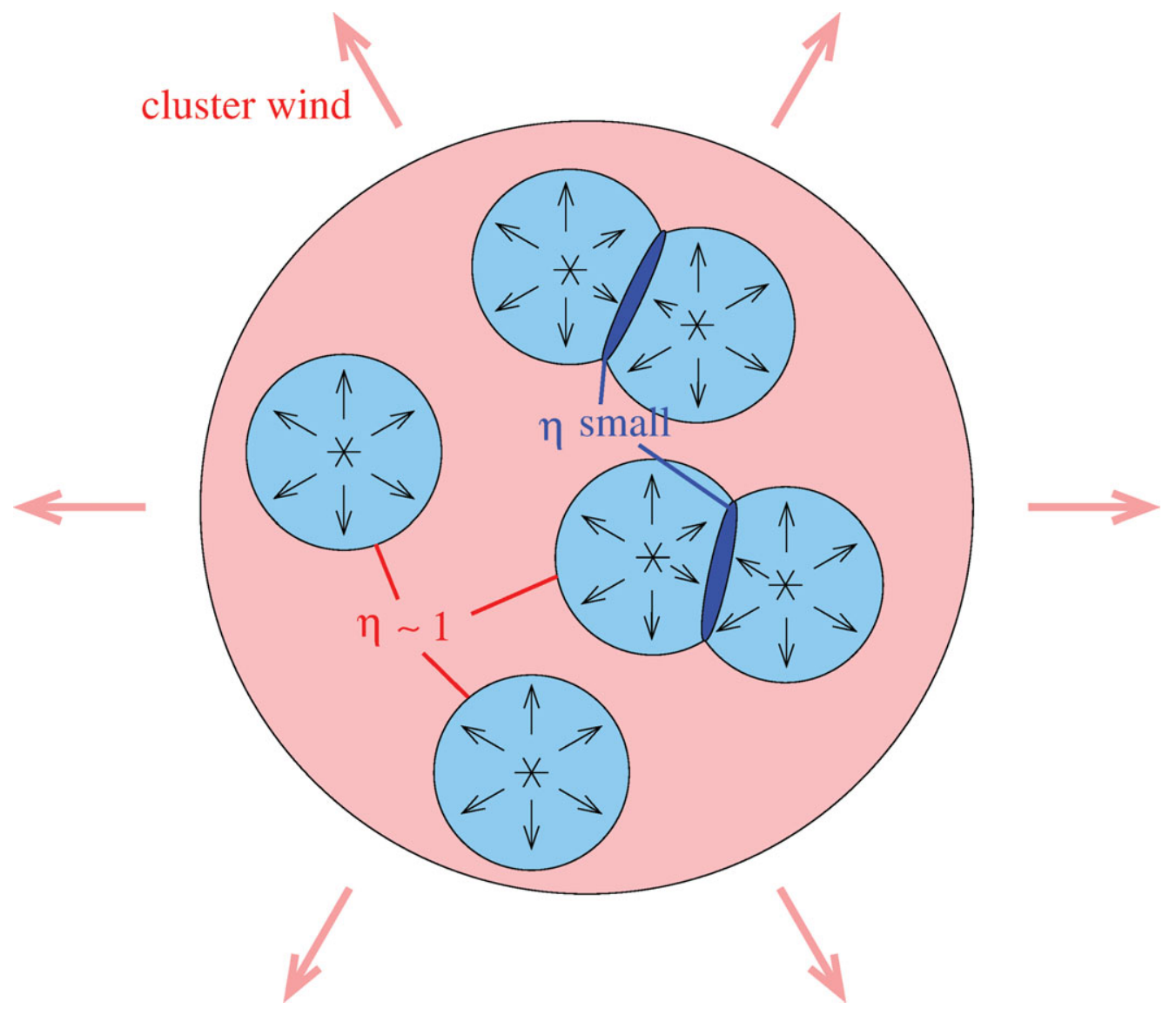

Figure 1. A model of the star cluster. Stars are homogeneously distributed in the cluster volume. The individual stellar winds collide and thermalize a fraction $\eta$ of their mechanical energy in shocks.

with an efficiency $\eta$. The value of $\eta$ is unknown; however, we try to estimate it from observations and also from numerical simulations. A schematic view of the modelled cluster is shown in Fig. 1.

In our model we give the solution of the hydrodynamical equations $(3.1-3.3)$. In some cases the external gravitational force is included:

$$
\begin{array}{r}
\frac{\partial \rho}{\partial t}+\nabla \cdot(\rho u)=q_{m} \\
\frac{\partial u}{\partial t}+(u \cdot \nabla) u+\nabla P / \rho=-\nabla \phi_{\mathrm{BH}+\mathrm{NSB}} \\
\frac{\partial e}{\partial t}+\nabla \cdot(e u)+P \nabla u=q_{e}-Q
\end{array}
$$

where $q_{m}$ and $q_{e}$ are the mass and energy deposition rates per unit of volume, $\rho$ is the density, $u$ is the velocity, and $e$ is the internal energy of the medium, $Q=n_{i} n_{e} \Lambda(T, Z)$ is the cooling rate, $n_{i}$ and $n_{e}$ are the ion and electron number densities, and $\Lambda(T, Z)$ is the Raymond and Cox (Plewa, 1995) cooling function, which depends on the thermalized gas temperature, $T$, and metalicity, $Z$. The right hand side of the equation (3.2) represents 
SFR

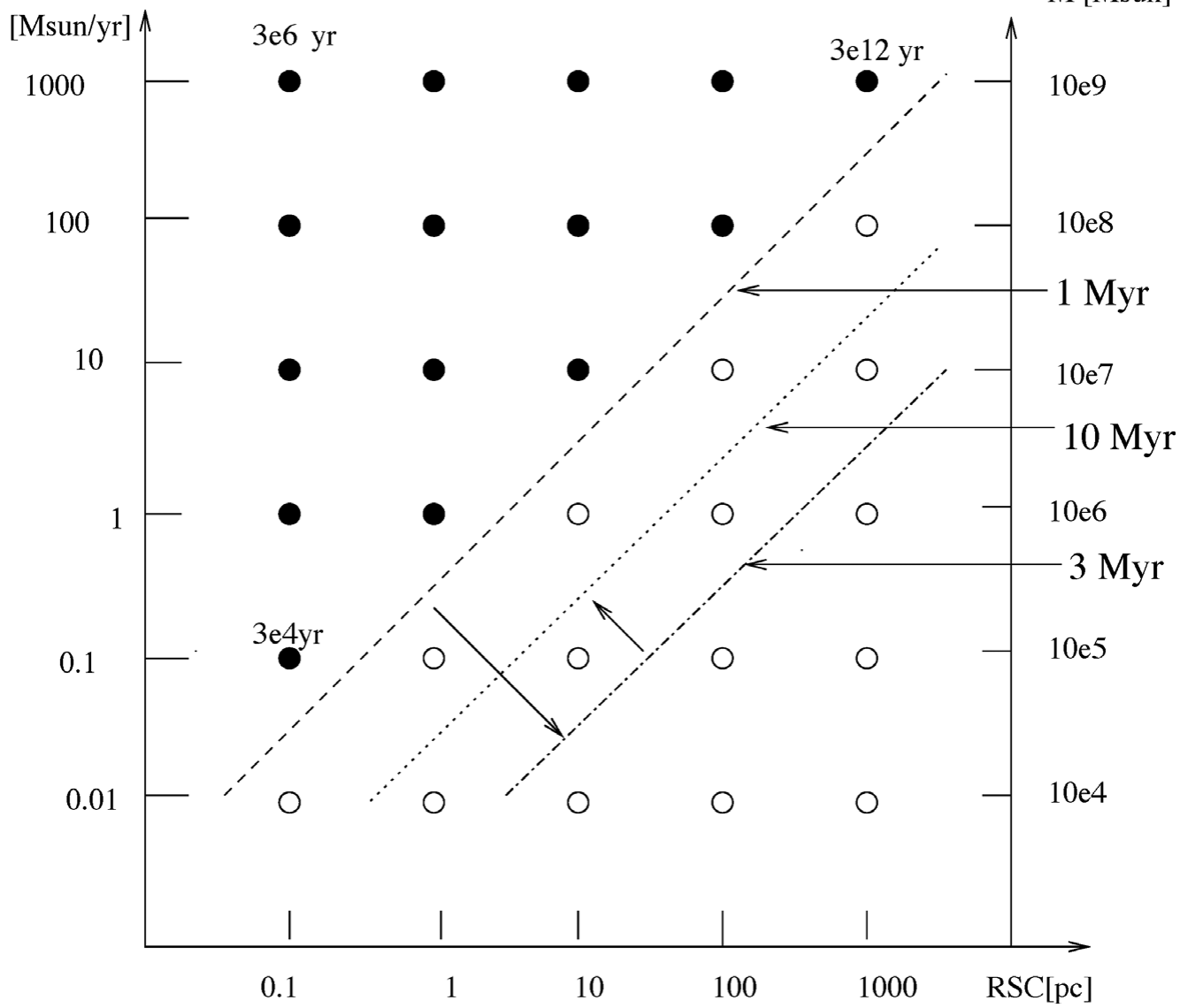

Figure 2. SFR versus $\mathrm{R}_{S C}$ plane for $\eta=0.01$. The positions of clusters at the age of $1 \mathrm{Myr}$ with the pure wind solutions (open circles) and bimodal solutions (filled circles) are given. The dashed line is the critical line dividing the pure wind area from the bimodal solution area. It changes its position with the age of the cluster as shown by arrows. The self-shielding time for a $10 \mathrm{Myr}$ old cluster is given for bimodal solutions at the extreme positions in the SFR - $\mathrm{R}_{S C}$ plane.

the external gravitational force of the central black hole and of the gravitating mass of the starburst itself. This external gravitational term has been considered in some cases only.

The adiabatic solution of hydrodynamical equations by Chevalier \& Clegg (1985) is valid when for certain cluster radii the mechanical wind energy is low enough. When we increase it above a certain critical value, the wind solution applies to the outer part of the cluster volume only. Inside of a stagnation radius $R_{s t}$, the intracluster medium is thermally unstable, forming warm clumps. The position of the critical line in the cluster mass versus cluster radius plane is very sensitive to the value of $\eta$ : small $\eta$ moves the critical line to smaller masses.

In Fig. 2 we show how the position of the critical line, in the case of $\eta=0.01$, changes its position during the first $10 \mathrm{Myr}$ of the cluster life. We assume that a cluster is formed during the first $1 \mathrm{Myr}$, and that its final mass is given by the star formation rate (SFR). 


\section{$*$}

\section{*}

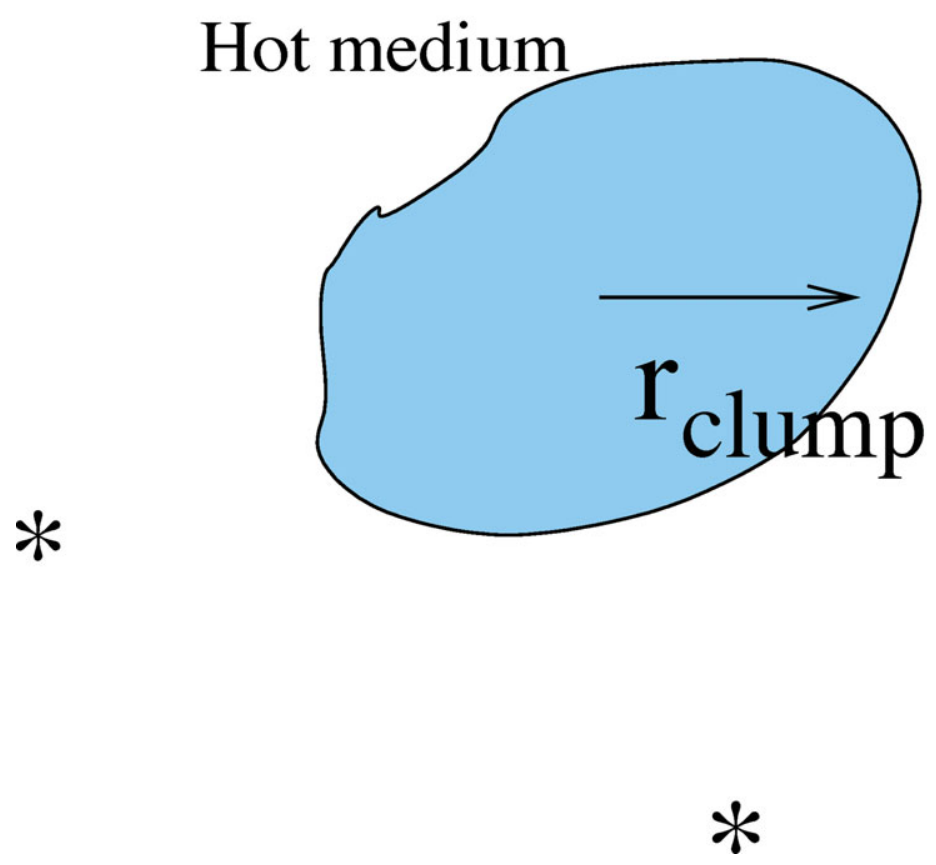

Figure 3. A warm clump inside of the thermally unstable central part of a forming cluster.

However, for larger values of $\eta$ the critical line shifts to the left upper corner of the SFR versus $\mathrm{R}$ plane. When $\eta$ is close to 1 , almost all the clusters are described by the single mode wind solution.

We are interested if such clumps (see Fig. 3) may be Jeans unstable. We assume that a warm clump is in pressure equilibrium in the hot medium, $n_{\text {clump }}=\frac{P_{h o t}}{k T}$, where $T=10^{4} \mathrm{~K}$ is the clump temperature. Thus the Jeans mass, $M_{\text {Jeans }}=2.5 \times$ $10^{5} M_{\odot}\left(\frac{n_{\text {clump }}}{10^{3} \mathrm{~cm}-3}\right)^{-3 / 2}$, depends sensitively on the pressure of the hot medium $P_{h o t}$. However, $P_{h o t}$ is very sensitive to the thermalization efficiency $\eta$. Low values of $\eta$ produce low values of $P_{h o t}$, which implies large $M_{\text {Jeans }}$. We conclude that if the value of $\eta$ is small, masses of warm clumps with the temperatures $10^{4} \mathrm{~K}$ have, after $10 \mathrm{Myr}$ of mass accumulation in the thermally unstable region of a young star cluster, masses much smaller than $M_{\text {Jeans }}$, and therefore they are gravitationally stable.

Another question is whether the thermally unstable clumps are able to self-shield against the UV photon field of the young star cluster. The time $t_{S S}$ of the clump growth needed to build clumps able to self-shield may be estimated with the following formula

$$
t_{S S}=\frac{3}{4} \mu m_{H}\left(\frac{k T}{P_{h o t}}\right)^{5}\left(\frac{3 \dot{N}_{U V, S C}}{4 \pi R_{S C} \alpha_{*}}\right)^{3}\left(\frac{N_{O B}}{\dot{M}_{S C}}\right),
$$

where $\alpha_{*}=1.58 \times 10^{-13} \mathrm{~cm}^{3} \mathrm{~s}^{-1}, \dot{N}_{U V, S C}$ is the number of UV photons produced by the star cluster, $\dot{M}_{S C}$ is the mass flux inserted by all the stars of the cluster and $N_{O B}$ is the 
number of OB stars. We assume that $N_{O B}$ also gives the number of warm clumps. A more detailed derivation of this will be given elsewhere (Palouš et al.(2010)).

The self-shielding times for $10 \mathrm{Myr}$ old clusters are given in Fig. 2. $t_{S S}$ is less than $1 \mathrm{Myr}$ for small clusters with radius less that $1 \mathrm{pc}$. There, the clumps are able to selfshield, which may enable the decrease of temperature in their central parts, and trigger secondary star formation there. On the other hand, in star forming regions larger that $10 \mathrm{pc}, t_{S S}$ is larger that $100 \mathrm{Myr}$, which probably eliminates the warm clumps as seeds of secondary star formation.

\section{Conclusions}

Outside of star clusters, the common action of the feedback of many stars forms shells, which may become gravitationally unstable, forming second generations of stars. We discuss the analytical and numerical models of expanding shells and analyze the mass spectra of fragments.

Inside of star clusters the bimodal wind solution applies above the critical line in the cluster mass versus radius plane. For small values of the thermalization efficiency, the warm clumps seem to be gravitationally stable, however, for clusters less that $1 \mathrm{pc}$ in radius the self-shielding time is short. The cores of warm clumps may further decrease their temperature, becoming seeds of secondary star formation.

\section{Acknowledgements}

The authors thank the referee for careful reading of the paper. The authors gratefully acknowledge the support by the Institutional Research Plan AV0Z10030501 of the Academy of Sciences of the Czech Republic and by the project LC06014 Center for Theoretical Astrophysics of the Ministry of Education, Youth and Sports of the Czech Republic. This study has been supported by CONACYT-México research grant 60333 and 47534-F. JD acknowledges support from a Marie Curie fellowship as part of the European Commission FP6 Research Training Network 'Constellation' under contract MRTN-CT-2006-035890.

\section{References}

Chevalier, R. A. \& Clegg, A. W. 1985, Nature, 317, 44

Dale, J., Wünsch, R., Whitworth, A., \& Palouš, J. 2009, MNRAS, 398, 1537

Dale, J. Wünsch, R. Smith, R. J., Whitworth, A., \& Palouš, J 2010, MNRAS, submitted

Deharveng, L., Lefloch, B., Zavagno, A., Caplan, J., Whitworth, A. P., Nadeau, D., \& Martín, S. $A \& A, 408, \mathrm{~L} 25$

Deharveng, L., Zavagno, A., Schuller, F., Caplan, J., Pomarès, M., \& De Breuck, C. 2009, A\&A, 496, 177

Ehlerová, S., Palouš, J. 2005, A $₫ A, 437,101$

Elmegreen, B. G. \& Lada, C. J. 1977, ApJ, 214, 725

Elmegreen, B. G. 1994 ApJ, 427, 384

Hartmann, D. \& Burton, W. B. 1997, Atlas of Galactic Neutral Hydrogen, Cambridge University Press

Hueyotl-Zahuantitla, F., Tenorio-Tagle, G., Wünsch, R., Silich, S., \& Palouš, J. 2010, ApJ, 716, 324

McClure-Griffiths, N. M., Dickey, J. M., Gaensler, B.M., \& Green, A., J. 2002, ApJ, 578, 176

Palouš, J., Wünsch, R., Silich, S., \& Tenorio-Tagle, G. 2010 in preparation

Plewa, T. 1995, MNRAS, 275, 143

Sidorin, V. 2008 IR, optical and X-ray counterparts of HI shells in the Milky Way, Diploma Thesis, Charles University, Prague 
Silich, S., Tenorio-Tagle, G., Muñoz-Tuñon, C., Hueyotl-Zahuantiutla, F., Wünsch, R., \& Palouš, J. 2010, ApJ, 711, 25

Tenorio-Tagle, G., Wünsch, R., Silich, S., \& Palouš, J. 2007, ApJ, 658, 1196

Tenorio-Tagle, G., Wünsch, R., Silich, S., Muñoz-Tuñon, C., \& Palouš, J. 2010, ApJ, 708, 1621

Vishniac, E. T. 1983 ApJ, 274, 152

Walter, F. \& Brinks, E. 1999, AJ, 118, 273

Walter, F., Brinks, E., de Blok, W. J. G., Bigiel, F., Kennicutt, R. C. Jr., Thornley, M. D., \& Leroy, A. 2008, $A J, 136,2563$

Watson, C., Corn, T., Churchwell, E. B., Babler, B. L., Povich, M. S., Meade, M. R., \& Whitney, B. A. 2009 ApJ, 694, 546

Whitworth, A.P., Bhattal, A. S., Chapman, S. J., Disney, M. J., \& Turner, J. A. 1994 MNRAS, 268,1341

Wünsch, R., Silich, S., Palouš, J., \& Tenorio-Tagle, G. 2007, A\&A, 471, 579

Wunsch, R., Tenorio-Tagle, G., Palouš, J., \& Silich, S. 2008, ApJ, 683, 683

Wünsch, R., Dale, J., Palouš, J., \& Whitworth, A. P. 2010, MNRAS, in press

Zavagno, A., Deharveng, L., Comerón, F., Brand, J., Massi, F., Caplan, J., \& Russeil, D. 2008 $A \mathscr{E} A, 446,171$ 